\title{
Small RNAs: regulators of plant development and climate resilience
}

\author{
Ramanjulu Sunkar $^{1} \cdot$ M. Maheswari ${ }^{2} \cdot$ Supriyo Chakraborty $^{3}$
}

Received: 6 December 2017 / Accepted: 8 December 2017/Published online: 30 December 2017

(C) Indian Society for Plant Physiology 2017

The quest for food security coupled with the enhanced nutritional quality of food grains are fundamental aspects of agriculture. There is a growing concern on the impending climate change and the environmental challenges it is posing to agricultural production in general, and it is in this context, that enhancing climate resilience gains crucial importance. Plant adaptation to both biotic and abiotic stresses, are multifaceted. Regulation of gene expression by Small RNAs forms a significant part by which plants can counter the abiotic and biotic challenges. The discovery and manipulation of Small RNA is one of the few cutting-edge scientific disciplines, which is evident from the plethora of literature available on this subject in the recent years. The small RNA biology such as the miRNA biogenesis, sub-cellular transport, degradation and regulation of gene expression are currently being studied in diverse plant species to fully understand the roles of these small RNAs in various biological processes.

It is in this context, that the Indian Journal of Plant Physiology considered it apt to come up with this year's special issue in the area of Small RNA with an effort to bring out the state-of-the-art in all aspects of Small RNA and its role in plants. This special issue focuses on the most

M. Maheswari

mmandapaka59@gmail.com

1 Department of Biochemistry and Molecular Biology, Oklahoma State University - Stillwater Oklahoma State, Stillwater, OK, USA

2 Division of Crop Sciences, ICAR-Central Research Institute for Dryland Agriculture, Hyderabad, Telangana 500059 , India

3 Molecular Virology Laboratory, School of Life Science, Jawaharlal Nehru University, New Delhi 110067, India often voiced thought on Small RNA bringing out the paradox "Small RNAs are big players in Plants". We trust that this is a very useful attempt in highlighting the big role of Small RNA in plants.

This special issue of Indian Journal of Plant Physiology has a diverse set of articles covering the basic as well as applied aspects of Small RNAs. The issue contains 16 review articles and five original research articles devoted to various aspects of small RNAs, which were contributed by more than 70 authors from globally reputed institutes and universities. As a result, this issue covers the broad scope and dynamics of studies on role of small RNAs in plant development, metabolism as well as response to biotic and abiotic stresses. Also an attempt has been made to review the status of research on small RNAs in a few crops such as wheat, potato, sorghum and rubber.

The aspects of miRNA bio-genesis and their role in leaf and root development have been described in 'The key role of small RNAs in the making of a leaf' and 'Role of miRNAs in root development in model plant Arabidopsis thaliana'. The major challenges of genome editing with miRNAs as targets for improvement of agriculturally important traits have been emphasized in 'micro RNAs: potential target for genome editing in plants for trait improvement'; and 'Micro-RNAs as targets for engineering biofuel feedstock sorghum'. The siRNA-mediated regulation of gene expression and its utilization as a potential reverse genetic tool for functional genomics studies in plants has been briefly described in 'small interfering RNA-mediated regulation of gene expression and its role as a plant reverse genetic tool'. Likewise, si-RNA dependent link between post transcriptional gene silencing (PTGS) and transcriptional gene silencing (TGS) have been addressed in 'Interplay and transition between small RNA 
directed post transcriptional and transcriptional gene silencing in plants'.

Role of small RNAs in enhancing tolerance to abiotic stresses, such as, drought, heat, and salinity have been discussed in articles on, 'Biomass production and salinity response in plants : role of micro RNAs'; 'sRNA and epigenetic mediated abiotic stress tolerance in plants'; 'small RNA and drought tolerance in plants' and 'Mirador on the potential role of miRNAs in synergy of light and heat networks'; 'Leaf wax trait in crops for drought and biotic stress tolerance : regulators of epicuticular wax synthesis and role of small RNAs'; Similarly, the role of small RNAs in biotic stress tolerance has been highlighted in articles 'Does endogenous RNAi have anti-pathogenic features ?'; 'Overview of plant RNA polymerases in antiviral defense and gene silencing'; 'Possible role of miRNAs and their targets in modulating leaf morphology and plant growth during leaf curl virus infection in tomato'.

The three original research articles are on the role of microRNAs in developmental processes as well as target gene expression in response to abiotic and biotic stresses. The article on 'MicroRNA expression profiles in the emerging tillers and inflorescence of switchgrass' revealed the identification of conserved as well as novel miRNA families which are a valuable resource to obtain insight into their role in tillering. A novel miRNA mi R430 was identified, cloned and mapped it on chromosome $3 \mathrm{~B}$ from wheat and the expression analysis suggested association with heat shock proteins in 'miR430: The Novel heat responsive microRNA'. 'In planta silencing of NSs and Hc-Pro through RNAi constructs' presents results suggesting that artificial-miRNA strategy is an effective and predictable approach to silence Groundnut bud necrosis virus and Papaya ring spot virus.

As guest editors of this Special issue of the Indian Journal of Plant Physiology, we place on record our thanks and gratitude to all the reviewers for their valuable contributions and constant support for reviewing these manuscripts on time. Many of them reviewed multiple manuscripts and their generous effort has made it possible to edit/revise and to publish this Special issue in a relatively short time.

Editors trust this special issue will be an exciting stimulus to researchers who are working in this area and also will help in showing new direction in the basic research to develop strategies for climate resilient agriculture. 